\title{
NON-COMMUTATIVE SCHUR-HORN THEOREMS AND EXTENDED MAJORIZATION FOR HERMITIAN MATRICES
}

\author{
PEDRO G. MASSEY \\ DEPARTAMENTO DE MATEMÁTICA - FCE, UNLP \& \\ INSTITUTO ARGENTINO DE MATEMÁTICA.
}

A Marina, con amor

\begin{abstract}
Let $\mathcal{A} \subseteq \mathcal{M}_{n}(\mathbb{C})$ be a unital $*$-subalgebra of the algebra $\mathcal{M}_{n}(\mathbb{C})$ of all $n \times n$ complex matrices and let $B$ be an hermitian matrix. Let $\mathcal{U}_{n}(B)$ denote the unitary orbit of $B$ in $\mathcal{M}_{n}(\mathbb{C})$ and let $\mathcal{E}_{\mathcal{A}}$ denote the trace preserving conditional expectation onto $\mathcal{A}$. We give a spectral characterization of the set

$$
\mathcal{E}_{\mathcal{A}}\left(\mathcal{U}_{n}(B)\right)=\left\{\mathcal{E}_{\mathcal{A}}\left(U^{*} B U\right): U \in \mathcal{M}_{n}(\mathbb{C}) \text {, unitary matrix }\right\} .
$$

We obtain a similar result for the contractive orbit of a positive semi-definite matrix $B$. We then use these results to extend the notions of majorization and submajorization between self-adjoint matrices to spectral relations that come together with extended (non-commutative) Schur-Horn type theorems.
\end{abstract}

Keywords. Extended majorization, non-commutative Schur-Horn theorems, diagonal block compressions, partial traces, unitary orbit.

2000 AMS Subject Classification: Primary 15A24, 15A42.

\section{INTRODUCTION}

The Schur-Horn theorem states $([15,27])$, roughly speaking, that the necessary and sufficient conditions on two vectors $x, y \in \mathbb{R}^{n}$ for the existence of an hermitian matrix $A$ with spectrum (counting multiplicities) $y$ and main diagonal $x$ are a finite number of linear inequalities involving the entries of $x$ and $y$. This result was the starting point for the work of Konstant [19] on actions of compact Lie groups that was subsequently extended to torus actions on symplectic manifolds by Atiyah [6], and Guillemin and Sternberg [14] independently. Recently, there has been interest in some geometric aspects of the original result of Schur and Horn [20] which turn out to have also implications in frame theory [24].

There have also been extensions of the Schur-Horn theorem to infinite dimension such as Neuman's work [25] on approximate diagonals of self-adjoint operators in $L(\mathcal{H})$, the work of Kadison $[16,17]$ particularly on diagonals of projections in $L(\mathcal{H})$, and the recent work of Arveson and Kadison [5] on diagonals of trace class operators, where they also focus on a possible extension of the Schur-Horn theorem to $\mathrm{II}_{1}$ factors. A weak version of the Arveson-Kadison conjecture is proved in [4]. Indeed, this exposition in strongly influenced by the point of view of [16] and [5] of the Schur-Horn theorem.

In [21] C.K. Li and Y.T. Poon obtained an extension of the Schur-Horn theorem, but in a different way. They found necessary and sufficient spectral conditions on two $n \times n$ self-adjoint matrices $A, B$ for the existence of an $n \times n$ unitary matrix $U$ such that $A$ is the block diagonal compression of $U^{*} B U$ with respect to certain block decomposition of $U^{*} B U$. Notice that the Schur-Horn theorem can be seen as a particular case of this problem, namely when the block representation of $U^{*} B U$ is with respect to $1 \times 1$ blocks. They showed that the situation with these general block diagonal compressions is quite different from that of the classical Schur-Horn theorem. The nature and the complexity of the necessary and sufficient spectral conditions they found are related with Horn-Klyachko's compatibility inequalities [18], which give necessary and

Partially supported by a pdf from PIMS, CONICET (PIP 4463/96), Universidad de La Plata (UNLP 11 X472) and ANPCYT (PICT03-09521).

Postal address: Saavedra $15,3^{\circ}$ piso (1083) Buenos Aires - Argentina

Email: massey@mate.unlp.edu.ar. 
sufficient conditions on $(m+1)$ vectors $\lambda^{i} \in \mathbb{R}^{n}, 0 \leq i \leq m$ for the existence of $(m+1) n \times n$ self-adjoint matrices $A_{i}$ with spectrum $\lambda^{i}$ for $0 \leq i \leq m$ and $A_{0}=A_{1}+\ldots+A_{m}$.

Notice that the block diagonal compression with respect to a fixed block decomposition of $n \times n$ matrices coincides with the (unique) conditional expectation onto the corresponding block diagonal (unital, self-adjoint) subalgebra, that preserves the trace. Thus, it seems natural to consider the following problem: given an arbitrary unital self-adjoint subalgebra $\mathcal{A}$ of the complex algebra of $n \times n$ matrices and two $n \times n$ self-adjoint matrices $A$ and $B$, find necessary and sufficient spectral conditions for the existence of a $n \times n$ unitary matrix $U$ such that

$$
\mathcal{E}_{\mathcal{A}}\left(U^{*} B U\right)=A,
$$

where $\mathcal{E}_{\mathcal{A}}$ denotes the unique conditional expectation onto $\mathcal{A}$ that preserves the trace. In this note we consider a systematic analysis of this and related problems, that can be considered as non commutative Schur-Horn type theorems (in the sense of [5]). Our main result regarding these problems is Theorem 3.6. Our approach is based on the work of Friedland [11] and Fulton [12] that extend that of Klyachko [18] on the spectrum of the sum of hermitian operators.

This finite dimensional operator algebra point of view is developed to introduce an extension of majorization between self-adjoint matrices as defined by Ando [1] to that of extended majorization between self-adjoint matrices. Briefly, given two self-adjoint $n \times n$ complex matrices $A$ and $B$, and $\mathbf{l}=(d(i), c(i))_{i=1}^{m} \in\left(\mathbb{N}^{2}\right)^{m}$ such that $\sum_{i=1}^{m} d(i) \cdot c(i)=n$ we say that $B \mathbf{l}$-majorizes $A$, and write $A \prec_{1} B$, if

$$
A=V\left(\mathcal{E}_{\mathcal{A}}\left(U^{*} B U\right)\right) V^{*} \text { for some } n \times n \text { unitaries } U, V
$$

where $\mathcal{A}=\oplus_{i=1}^{m} \mathcal{M}_{d(i)}(\mathbb{C}) \otimes 1_{c(i)} \subseteq \mathcal{M}_{n}(\mathbb{C})$ and $\mathcal{E}_{\mathcal{A}}$ denotes the trace preserving conditional expectation onto $\mathcal{A}$ (for an explicit description of $\mathcal{E}_{\mathcal{A}}$ see Eq. (7)). By the classical Schur-Horn theorem we can see that if $\mathbf{l}=(1,1)_{i=1}^{n}$ then the extended majorization relation $A \prec_{\mathbf{l}} B$ is the same as the majorization $A \prec B$ in the sense of Ando.

We also consider the relation of extended majorization with some convex functionals. As in the case of usual majorization, the notion of extended majorization has relations with "signal processing" ([3, 10, 24]), but it seems that in this case the word "quantum" may be added ([23]). As an example of this last claim, we obtain a result related with a conjecture posed by M.B. Ruskai and K. Audenaert in Quantum Information Theory (QIT).

\section{Preliminaries}

Some notations and terminology. We denote by $\mathcal{M}_{n}(\mathbb{C})\left(\operatorname{resp} . \mathcal{M}_{n}(\mathbb{C})^{s a}, \mathcal{M}_{n}(\mathbb{C})^{+}, \mathcal{U}(n)\right)$ the set of $n \times n$ complex (resp self-adjoint, positive semi-definite, unitary) matrices, with identity $1_{n}$. By a system of projections $\mathcal{P}=\left\{P_{i}\right\}_{i=1}^{m}$ in $\mathcal{M}_{n}(\mathbb{C})$ we mean an ordered set of $n \times n$ complex orthogonal projection matrices such that $\sum_{i=1}^{m} P_{i}=1_{n}$. Given a system of projections $\mathcal{P}=\left\{P_{i}\right\}_{i=1}^{m}$ in $\mathcal{M}_{n}(\mathbb{C})$ we consider the compression $\mathcal{C}_{\mathcal{P}}: \mathcal{M}_{n}(\mathbb{C}) \rightarrow \mathcal{M}_{n}(\mathbb{C})$ induced by $\mathcal{P}$ given by $\mathcal{C}_{\mathcal{P}}(S)=\sum_{i=1}^{m} P_{i} S P_{i}$. Notice that $\mathcal{C}_{\mathcal{P}}$ is a trace preserving completely positive map. If $(c(i), d(i))_{i=1}^{m} \in\left(\mathbb{N}^{2}\right)^{m}$ is such that $\sum_{i=1}^{m} c(i) \cdot d(i)=n$ then we shall consider $\oplus_{i=1}^{m} \mathcal{M}_{d(i)}(\mathbb{C}) \otimes 1_{c(i)} \subseteq \mathcal{M}_{n}(\mathbb{C})$ as a unital $*$-subalgebra of $\mathcal{M}_{n}(\mathbb{C})$. If $x \in \mathbb{R}^{n}$ then we denote by $x^{\downarrow} \in \mathbb{R}^{n}$ the vector obtained from $x$ by rearranging the coordinates of $x$ in non-increasing order. If $A \in \mathcal{M}_{n}(\mathbb{C})^{s a}$ then $\lambda(A)=\lambda(A)^{\downarrow} \in \mathbb{R}^{n}$ denotes the $n$-tuple of eigenvalues of $A$ counting multiplicities and arranged in non-increasing order. If $S \in \mathcal{M}_{n}(\mathbb{C})$ then $\mathcal{U}_{n}(S), \mathcal{C}_{n}(S)$ denote respectively the unitary and contractive orbit of $S$ i.e. $\mathcal{U}_{n}(S)=\left\{U^{*} S U: U \in \mathcal{U}(n)\right\}, \mathcal{C}_{n}(S)=\left\{V^{*} S V: V \in \mathcal{M}_{n}(\mathbb{C}),\|V\| \leq 1\right\}$. More generally, $\mathcal{U}_{n}(\mathcal{X}), \mathcal{C}_{n}(\mathcal{X})$ denote the unitary and contractive orbit of $\mathcal{X} \subseteq \mathcal{M}_{n}(\mathbb{C})$. We shall denote the canonical basis of $\mathbb{C}^{n}$ as $\left\{e_{i}\right\}_{i=1}^{n}$. If $\lambda \in \mathbb{R}^{n}$ we denote by $\operatorname{Diag}(\lambda)$ the diagonal matrix with main diagonal $\lambda$. The set $\{1, \ldots, n\}$ is denoted by $\langle n\rangle$. We denote by $\mathbb{R}_{\geq 0}$ the set of non-negative real numbers.

2.1. Horn-Klyachko's theory on sums of hermitian matrices. We briefly describe some basic notions of Schubert varieties and admissible $m$-tuples to state Theorem 2.1. This result summarizes the deep work of Klyachko [18], Friedland [11] and Fulton [12]. For a detailed account on these and related topics we refer the reader to [13] and the references therein. 
Let $V_{*}=V_{1} \subset V_{2} \subset \cdots V_{n}=\mathbb{C}^{n}$ be a complete flag on $\mathbb{C}^{n}$ i.e. $\operatorname{dim}\left(V_{i}\right)=i$ for $1 \leq i \leq n$. Fix $1 \leq r<n$ and let $I=\left\{i_{1}, \ldots, i_{r}\right\} \subset\langle n\rangle$ with $1 \leq i_{1}<i_{2}<\cdots<i_{r} \leq n$. Denote

$$
I^{\prime}=\left\{i_{1}^{\prime}, \ldots, i_{r}^{\prime}\right\}, \quad i_{j}^{\prime}=n+1-i_{r+1-j}, \quad j=1, \ldots, r .
$$

Let $X=G r\left(r, \mathbb{C}^{n}\right)$ be the Grassmann variety of all $r$-dimensional subspaces $L$ of $\mathbb{C}^{n}$. Let $\Omega_{I}\left(V_{*}\right)$ be the Schubert variety in $X$ defined by

$$
\Omega_{I}\left(V_{*}\right):=\left\{L \in X: \operatorname{dim}\left(L \cap V_{i_{j}}\right) \geq j \text { for } 1 \leq j \leq r\right\} .
$$

An $(m+1)$-tuple $\left(I_{0}, \ldots, I_{m}\right)$ of subsets $I_{0}, \ldots, I_{m}$ of $\langle n\rangle$, each of cardinality $r(1 \leq r<n)$ is called admissible, if for any $(m+1)$ complete flags $V_{*}^{0}, \ldots, V_{*}^{m}$ of $\mathbb{C}^{n}$ the following condition holds:

$$
\Omega_{I_{0}}\left(V_{*}^{0}\right) \cap\left(\bigcap_{j=1}^{m} \Omega_{I_{j}^{\prime}}\left(V_{*}^{j}\right)\right) \neq \emptyset .
$$

We will use the following notations. Let $|J|$ denote the cardinal of the set $J$ and let

$$
x[I]:=\sum_{i \in I} x_{i}, \quad x \in \mathbb{R}^{n}, \quad I \subseteq\langle n\rangle, \quad|I| \geq 1 .
$$

Theorem $2.1([18,11,12])$. Let $\left(\lambda^{i}\right)_{i=0}^{m} \in\left(\mathbb{R}^{n}\right)^{(m+1)}$ be an $(m+1)$-tuple of vectors in $\mathbb{R}^{n}$. Then

(i) There exist $m+1$ matrices $A_{0}, \ldots, A_{m} \in \mathcal{M}_{n}(\mathbb{C})^{\text {sa }}$ such that $\lambda\left(A_{i}\right)=\lambda^{i}$ for $0 \leq i \leq m$ and $A_{0}=\sum_{i=1}^{m} A_{i}$ if and only if $\lambda^{0}[\langle n\rangle]=\sum_{i=1}^{m} \lambda^{i}[\langle n\rangle]$ and

$$
\lambda^{0}\left[I_{0}^{\prime}\right] \geq \sum_{j=1}^{m} \lambda^{j}\left[I_{j}^{\prime}\right], \quad \text { for every admissible }(m+1) \text {-tuple }\left(I_{j}\right)_{j=0}^{m} \text {. }
$$

(ii) There exist $m+1$ matrices $A_{0}, \ldots, A_{m} \in \mathcal{M}_{n}(\mathbb{C})^{\text {sa }}$ such that $\lambda\left(A_{i}\right)=\lambda^{i}$ for $0 \leq i \leq m$ and $A_{0} \geq \sum_{i=1}^{m} A_{i}$ if and only if $\lambda^{0}[\langle n\rangle] \geq \sum_{i=1}^{m} \lambda^{i}[\langle n\rangle]$ and the inequalities (2) hold.

We point out that the inequalities in (2) are rather the dual inequalities to those that appear in $[11,12,18]$. The fact that the theorem above follows from those papers is a consequence of the following equalities: for $I=\left\{i_{1}, \ldots, i_{r}\right\} \subseteq\langle n\rangle$ as above and $\lambda \in \mathbb{R}^{n}$ such that $\lambda=\lambda^{\downarrow}$ then

$$
(-\lambda)^{\downarrow}[I]=\sum_{j=1}^{r}-\lambda_{n+1-i_{j}}=-\sum_{j=1}^{r} \lambda_{n+1-i_{r+1-j}}=-\left(\lambda\left[I^{\prime}\right]\right) .
$$

As noted in [11], (i) follows from (ii). The inequalities in (2) are referred to as Horn-Klyachko's compatibility inequalities. We say that an $(m+1)$-tuple $\left(\lambda^{i}\right)_{i=0}^{m} \in\left(\mathbb{R}^{n}\right)^{(m+1)}$ satisfies HornKlyachko's compatibility inequalities if it satisfies the family of inequalities given in (2).

\section{Non commutative Schur-Horn theorems}

We say that $\left\{P_{i}\right\}_{i=1}^{m} \subseteq \mathcal{M}_{n}(\mathbb{C})$ is a system of coordinate projections if there exists a partition $\left\{\mathcal{J}_{i}\right\}_{i=1}^{m}$ of $\langle n\rangle$ by increasing subintervals (i.e. if $k_{1} \leq k \leq k_{2}$ with $k_{1}, k_{2} \in \mathcal{J}_{i}$ then $k \in \mathcal{J}_{i}$ for $1 \leq i \leq m$ and if $k \in \mathcal{J}_{i}, l \in \mathcal{J}_{j}$ then $k \leq l$ whenever $\left.i \leq j\right)$ such that $P_{i}$ is the projection onto $\operatorname{span}\left\{e_{k}, k \in \mathcal{J}_{i}\right\}$ for $1 \leq i \leq m$. Notice that in this case $\mathcal{C}_{\mathcal{P}}: \mathcal{M}_{n}(\mathbb{C}) \rightarrow \oplus_{i=1}^{m} \mathcal{M}_{d(i)}(\mathbb{C}) \subseteq \mathcal{M}_{n}(\mathbb{C})$, where $\operatorname{rank}\left(P_{i}\right)=d(i)$ for $1 \leq i \leq m$. If $\mathcal{Q}=\left\{Q_{i}\right\}_{i=1}^{m} \subseteq \mathcal{M}_{n}(\mathbb{C})$ is an arbitrary system of projections with $\operatorname{rank}\left(Q_{i}\right)=d(i)$ for $1 \leq i \leq m$ then there exists a unitary operator $W \in \mathcal{U}(n)$ such that $Q_{i}=W^{*} P_{i} W$ for $1 \leq i \leq m$ and hence $\mathcal{C}_{\mathcal{Q}}(X)=W^{*} \mathcal{C}_{\mathcal{P}}\left(W X W^{*}\right) W$ for $X \in \mathcal{M}_{n}(\mathbb{C})$. Hence, these coordinate systems of projections are a model for more general systems of projections.

With the previous terminology, we recall the classical Schur-Horn theorem. To that end, recall that given $x, y \in \mathbb{R}^{n}$ then we say that $x$ is submajorized by $y$, denoted $x \prec_{w} y$, if for $1 \leq k \leq n$ then $\sum_{i=1}^{k} x_{i}^{\downarrow} \leq \sum_{i=1}^{k} y_{i}^{\downarrow}$. If $x \prec_{w} y$ and moreover $\sum_{i=1}^{n} x_{i}=\sum_{i=1}^{n} y_{i}$, we say that $x$ is majorized by $y$ and write $x \prec y$.

Theorem 3.1 (Schur - Horn). Let $\mathcal{P}=\left\{P_{i}\right\}_{i=1}^{n} \subset \mathcal{M}_{n}(\mathbb{C})$ be the system of coordinate projections such that $\operatorname{rank}\left(P_{i}\right)=1,1 \leq i \leq n$. If $S \in \mathcal{M}_{n}(\mathbb{C})^{\text {sa }}$ then

$$
\left\{\mathcal{C}_{\mathcal{P}}\left(U^{*} S U\right): U \in \mathcal{U}(n)\right\}=\left\{\operatorname{Diag}(x): x \in \mathbb{R}^{n}, x \prec \lambda(S)\right\} .
$$

In what follows we denote by $\mathbf{e}^{d} \in \mathbb{R}^{d}$ the vector with all coordinates equal to 1 . 
Lemma $3.2([21])$. Let $\mathcal{P}=\left\{P_{i}\right\}_{i=1}^{m} \subseteq \mathcal{M}_{n}(\mathbb{C})$ be a system of coordinate projections in $\mathcal{M}_{n}(\mathbb{C})$ with $\operatorname{rank}\left(P_{i}\right)=d(i)$ and let $\mathcal{C}_{\mathcal{P}}: \mathcal{M}_{n}(\mathbb{C}) \rightarrow \oplus_{i=1}^{m} \mathcal{M}_{d(i)}(\mathbb{C})$ be the compression induced by $\mathcal{P}$. Let $S \in \mathcal{M}_{n}(\mathbb{C})^{s a}$ and $S_{i} \in \mathcal{M}_{d(i)}(\mathbb{C})^{s a}$ be such that $\lambda\left(S_{i}\right)=\lambda^{i} \in \mathbb{R}^{d(i)}$ for $1 \leq i \leq m$ and let $\alpha \in \mathbb{R}$ be such that $S+\alpha 1_{n} \in \mathcal{M}_{n}(\mathbb{C})^{+}$. Then the following statements are equivalent:

(i) There exists a unitary matrix $U \in \mathcal{U}(n)$ such that

$$
\mathcal{C}_{\mathcal{P}}\left(U^{*} S U\right)=\oplus_{i=1}^{m} S_{i}
$$

(ii) There exist unitary matrices $U_{i} \in \mathcal{U}(n)$ for $1 \leq i \leq m$ such that

$$
S+\alpha 1_{n}=\sum_{i=1}^{m} U_{i}^{*}\left(\oplus_{j=1}^{m} \delta_{i j}\left(S_{i}+\alpha 1_{d(i)}\right)\right) U_{i} .
$$

(iii) The $(m+1)$-tuple

$$
\left(\lambda(S)+\alpha \mathbf{e}^{n},\left(\lambda^{1}+\alpha \mathbf{e}^{d_{1}}, 0_{n-d(1)}\right), \ldots,\left(\lambda^{m}+\alpha \mathbf{e}^{d_{m}}, 0_{n-d(m)}\right)\right) \in\left(\mathbb{R}_{\geq 0}^{n}\right)^{(m+1)}
$$

and satisfies Horn-Klyachko's compatibility inequalities plus $\operatorname{tr}(S)=\sum_{i=1}^{m} \operatorname{tr}\left(S_{i}\right)$.

Remark 3.3. Using Lemma $3.2(\alpha=0)$ and the classical Schur-Horn Theorem 3.1, we can see that if $\mathbf{a}=\left(a_{1}, \ldots, a_{n}\right), \mathbf{b}=\left(b_{1}, \ldots, b_{n}\right) \in\left(\mathbb{R}_{\geq 0}\right)^{n}$ then the $(n+1)$-tuple $\left(\mathbf{b}, a_{1} \cdot e_{1}, \ldots, a_{n} \cdot e_{1}\right)$ satisfies Horn-Klyachko's compatibility inequalities together with $\sum_{i=1}^{n} a_{i}=\sum_{i=1}^{n} \lambda_{i}$ if and only if $\mathbf{a} \prec \mathbf{b}$.

This last fact suggests that there might be alternative sets of linear inequalities for the spectral conditions in Lemma 3.2, which are less complex than Horn-Klyachko's compatibility inequalities. Such a reduction of the complexity of this problem has been done in [21, Thm 3.3]. They find a reduced set of the set of Horn-Klyachko's inequalities to be checked in order that the $(m+1)$ tuple $\left(\lambda,\left(\lambda_{1}, 0_{n-d(1)}\right), \ldots\left(\lambda_{m}, 0_{n-d(m)}\right)\right)$ satisfies all of Horn-Klyachko's inequalities. They show that the complexity of this reduced set actually depends on the dimensions $d(1), \ldots, d(m)$.

One of the most important consequences of the Schur-Horn theorem as stated in (3), is the fact that the left-hand side of that equality is a convex set (because the right-hand side is easily seen to be convex). It turns out that this is a particular feature of the diagonal compression $\mathcal{E}_{\mathcal{D}}$ onto a maximal abelian $*$-subalgebra of $\mathcal{M}_{n}(\mathbb{C})$. Indeed, let $\mathcal{P}=\left\{P_{i}\right\}_{i=1}^{m} \subseteq \mathcal{M}_{n}(\mathbb{C})$ be a system of coordinate projections in $\mathcal{M}_{n}(\mathbb{C})$ with $\operatorname{rank}\left(P_{i}\right)=d(i)$ and let $\mathcal{C}_{\mathcal{P}}: \mathcal{M}_{n}(\mathbb{C}) \rightarrow \oplus_{i=1}^{m} \mathcal{M}_{d(i)}(\mathbb{C})$ be the compression induced by $\mathcal{P}$. Assume, without loss of generality, that $d(1) \geq 2$. We define

$$
S=\left(\begin{array}{ll}
2 & 0 \\
0 & 4
\end{array}\right) \oplus 0_{(n-2)} \quad \text { and } \quad V=\left(\begin{array}{ll}
0 & 1 \\
1 & 0
\end{array}\right) \oplus 1_{(n-2)} .
$$

In this case

$$
T:=\frac{1}{2}\left(\mathcal{C}_{\mathcal{P}}(S)+\mathcal{C}_{\mathcal{P}}\left(V^{*} S V\right)\right)=\frac{1}{2}\left(S+V^{*} S V\right)=\left(\begin{array}{ll}
3 & 0 \\
0 & 3
\end{array}\right) \oplus 0_{(n-2)} .
$$

Assume now that there exists $U \in \mathcal{U}(n)$ such that $\mathcal{C}_{\mathcal{P}}\left(U^{*} S U\right)=T$. But, since $U^{*} S U \geq 0$ and $d(1) \geq 2$, the equality above implies that $U^{*} S U=T$. This last fact is a contradiction, since these two matrices have different spectrum.

The following result complements Lemma 3.2; since its proof can be adapted from that of Lemma 3.2 (see [21]) we omit it.

Lemma 3.4. Let $\mathcal{P}=\left\{P_{i}\right\}_{i=1}^{m} \subseteq \mathcal{M}_{n}(\mathbb{C})$ be a system of coordinate projections with rank $\left(P_{i}\right)=d(i)$ and let $\mathcal{C}_{\mathcal{P}}: \mathcal{M}_{n}(\mathbb{C}) \rightarrow \oplus_{i=1}^{m} \mathcal{M}_{d(i)}(\mathbb{C})$ be the compression induced by $\mathcal{P}$. If $S \in \mathcal{M}_{n}(\mathbb{C})^{+}$and $S_{i} \in \mathcal{M}_{d(i)}(\mathbb{C})^{+}$are such that $\lambda\left(S_{i}\right)=\lambda^{i} \in\left(\mathbb{R}_{\geq 0}\right)^{d(i)}$ for $1 \leq i \leq m$, then the following statements are equivalent:

(i) There exists a contraction $V \in \mathcal{M}_{n}(\mathbb{C})$ such that

$$
\mathcal{C}_{\mathcal{P}}\left(V^{*} S V\right)=\oplus_{i=1}^{m} S_{i}
$$

(ii) There exist unitary matrices $U_{i} \in \mathcal{U}(n)$ for $1 \leq i \leq m$ such that

$$
S \geq \sum_{i=1}^{m} U_{i}^{*}\left(\oplus_{j=1}^{m} \delta_{i j} S_{j}\right) U_{i}
$$


(iii) There exist a contraction $W \in \mathcal{M}_{n}(\mathbb{C})$ and unitary matrices $V_{i} \in \mathcal{U}(n)$ for $1 \leq i \leq m$ such that

$$
W^{*} S W=\sum_{i=1}^{m} V_{i}^{*}\left(\oplus_{j=1}^{m} \delta_{i j} S_{j}\right) V_{i} .
$$

(iv) The $(m+1)$-tuple $\left(\lambda(S),\left(\lambda^{1}, 0_{n-d(1)}\right), \ldots,\left(\lambda^{m}, 0_{n-d(m)}\right)\right)$ satisfies Horn-Klyachko's compatibility inequalities plus $\operatorname{tr}(S) \geq \sum_{i=1}^{m} \operatorname{tr}\left(S_{i}\right)$.

We now recall some basic facts about unital $*$-subalgebras and trace preserving conditional expectations in $\mathcal{M}_{n}(\mathbb{C})$. Let $\mathcal{A} \subseteq \mathcal{M}_{n}(\mathbb{C})$ be a unital $*$-subalgebra. Then, $\mathcal{A}$ is a subspace of the finite dimensional complex inner product space $\left(\mathcal{M}_{n}(\mathbb{C}),\langle\cdot, \cdot\rangle_{\operatorname{tr}}\right)$ where $\langle A, B\rangle_{\operatorname{tr}}=\operatorname{tr}\left(B^{*} A\right)$. Thus, we can consider $\mathcal{E}_{\mathcal{A}}$ the orthogonal projection with respect to $\langle\cdot, \cdot\rangle_{\text {tr }}$ onto $\mathcal{A}$. That is, $\mathcal{E}_{\mathcal{A}}: \mathcal{M}_{n}(\mathbb{C}) \rightarrow \mathcal{M}_{n}(\mathbb{C})$ is a linear, $\mathcal{E}_{\mathcal{A}} \circ \mathcal{E}_{\mathcal{A}}=\mathcal{E}_{\mathcal{A}}$ and

$$
\operatorname{tr}\left(C^{*} \mathcal{E}_{\mathcal{A}}(B)\right)=\operatorname{tr}\left(\mathcal{E}_{\mathcal{A}}(C)^{*} B\right), \quad \mathcal{E}_{\mathcal{A}}(A)=A, \quad \forall A \in \mathcal{A} .
$$

In the operator algebra context $\mathcal{E}_{\mathcal{A}}$ is called the trace preserving conditional expectation (TCE) onto $\mathcal{A}$; the fact that it is trace preserving is a consequence of the relations in (5) setting $C=1$ and recalling that $1 \in \mathcal{A}$. The TCE is uniquely determined by the previous properties.

We consider first the following two examples. Let $\mathcal{P}=\left\{P_{i}\right\}_{i=1}^{m}$ be a system of coordinate projections with $\operatorname{rank}\left(P_{i}\right)=d(i)$ for $1 \leq i \leq m$, and consider $\mathcal{A}=\oplus_{i=1}^{m} \mathcal{M}_{d(i)}(\mathbb{C}) \subset \mathcal{M}_{n}(\mathbb{C})$. Then $\mathcal{A}$ is a unital $*$-subalgebra of $\mathcal{M}_{n}(\mathbb{C})$ and the compression $\mathcal{C}_{\mathcal{P}}=\mathcal{E}_{\mathcal{A}}$ is the TCE onto $\mathcal{A}$. For the second example, consider first the identification of $\mathcal{M}_{d}(\mathbb{C}) \otimes \mathcal{M}_{m}(\mathbb{C})$ with $\mathcal{M}_{d \cdot m}(\mathbb{C})$ given by

$$
A \otimes B \approx\left(b_{i j} A\right)_{i, j=1}^{m} .
$$

Then the algebra $\mathcal{M}_{d}(\mathbb{C}) \otimes 1_{m}$ regarded inside of $\mathcal{M}_{d \cdot m}(\mathbb{C})$ is a unital $*$-subalgebra of $\mathcal{M}_{d \cdot m}(\mathbb{C})$ (the algebra of $m \times m$ block diagonal matrices with equal diagonal blocks). In this case, we can describe the TCE onto $\mathcal{A}$ by $\mathcal{E}_{\mathcal{A}}(C)=\frac{1}{m} \operatorname{Tr}_{m}(C) \otimes 1_{m}$, where $\operatorname{Tr}_{m}(C)=\sum_{i=1}^{m} C_{i i} \in \mathcal{M}_{d}(\mathbb{C})$ is a partial trace (see [7] for an alternative description).

In general, a unital $*$-subalgebra of $\mathcal{M}_{n}(\mathbb{C})$ can be described, up to conjugation by a unitary matrix $U \in \mathcal{M}_{n}(\mathbb{C})$, as a direct sum of $m$ blocks, each of the form $M_{d(i)} \otimes 1_{c(i)}$ for $1 \leq i \leq$ $m$ and such that $\sum_{i=1}^{m} d(i) c(i)=n$. The list $(d(i), c(i))_{i=1}^{m}$, that we call the spectral list, is invariant under unitary conjugations. Moreover, two unital $*$-subalgebras $\mathcal{A}, \mathcal{B} \subset \mathcal{M}_{n}(\mathbb{C})$ with spectral lists $\left(d_{\mathcal{A}}(i), c_{\mathcal{A}}(i)\right)_{i=1}^{m}$ and $\left(d_{\mathcal{B}}(i), c_{\mathcal{B}}(i)\right)_{i=1}^{r}$ are unitary conjugate (i.e. there exists a unitary $U \in \mathcal{U}(n)$ with $U^{*} \mathcal{A} U=\mathcal{B}$ ) if and only if $m=r$ and there exists a permutation $\sigma \in \mathbb{S}_{m}$ such that $\left(d_{\mathcal{A}}(i), c_{\mathcal{A}}(i)\right)=\left(d_{\mathcal{B}}(\sigma(i)), c_{\mathcal{B}}(\sigma(i))\right)$ for $1 \leq i \leq m$. In this case we say that the lists $\left(d_{\mathcal{A}}(i), c_{\mathcal{A}}(i)\right)_{i=1}^{m}$ and $\left(d_{\mathcal{B}}(i), c_{\mathcal{B}}(i)\right)_{i=1}^{r}$ are equivalent. Strictly speaking, the spectral list of a unital $*$-subalgebra is defined only up to equivalence, but we shall allow this abuse of language as it will not cause any problems with the notions to be considered.

If the spectral list of a unital $*$-subalgebra $\mathcal{A}$ is multiplicity free i.e. it is of the form $(d(i), 1)_{i=1}^{m}$ then we say that $\mathcal{A}$ is multiplicity free. The multiplicity free algebras (lists) are in some sense the well-behaved algebras (lists) in our context.

Let $\mathcal{A}=\oplus_{i=1}^{m} \mathcal{M}_{d(i)} \otimes 1_{c(i)}$ be a unital $*$-subalgebra of $\mathcal{M}_{n}(\mathbb{C})$ with spectral list $(d(i), c(i))_{i=1}^{m}$ and let $\mathcal{P}=\left\{P_{i}\right\}_{i=1}^{m}$ be a system of coordinate projections with $\operatorname{rank}\left(P_{i}\right)=d(i) \cdot c(i)$. Then, the TCE onto $\mathcal{A}$ can be described in terms of block diagonal compressions and partial traces as

$$
\mathcal{E}_{\mathcal{A}}(B)=\oplus_{i=1}^{m} \frac{1}{c(i)} \operatorname{Tr}_{c(i)}\left(B_{i}\right) \otimes 1_{c(i)}, \quad \text { where } \quad \mathcal{C}_{\mathcal{P}}(B)=\oplus_{i=1}^{m} B_{i} .
$$

If $\mathcal{B}$ is a unital $*$-subalgebra with an equivalent spectral list to that of $\mathcal{A}$ then, as stated before, there exists a unitary $U \in \mathcal{U}(n)$ such that $U^{*} \mathcal{A} U=\mathcal{B}$, so

$$
\mathcal{E}_{\mathcal{B}}(C)=U^{*} \mathcal{E}_{\mathcal{A}}\left(U C U^{*}\right) U
$$

This last fact can be verified using the uniqueness of the TCE onto $\mathcal{B}$. In what follows, given $\mathcal{A}, \mathcal{X} \subseteq \mathcal{M}_{n}(\mathbb{C})$ with $\mathcal{A}$ a unital $*$-subalgebra and $\mathcal{X}$ an arbitrary set, we denote by $\mathcal{E}_{\mathcal{A}}(\mathcal{X})$ the set of all values $\mathcal{E}_{\mathcal{A}}(x)$ for $x \in \mathcal{X}$. The following result is an immediate consequence of (8).

Lemma 3.5. Let $\mathcal{A}, \mathcal{B}$ be $*$-subalgebras of $\mathcal{M}_{n}(\mathbb{C})$ with equivalent spectral lists. Then,

$$
\mathcal{U}_{n}\left(\mathcal{E}_{\mathcal{A}}\left(\mathcal{U}_{n}(S)\right)\right)=\mathcal{U}_{n}\left(\mathcal{E}_{\mathcal{B}}\left(\mathcal{U}_{n}(S)\right)\right) \quad \text { and } \quad \mathcal{U}_{n}\left(\mathcal{E}_{\mathcal{A}}\left(\mathcal{C}_{n}(S)\right)\right)=\mathcal{U}_{n}\left(\mathcal{E}_{\mathcal{B}}\left(\mathcal{C}_{n}(S)\right)\right) .
$$


We now state and prove the (finite dimensional operator algebra version of the) NC-SchurHorn theorem. In what follows, if $\lambda^{i} \in \mathbb{R}^{d(i)}$ for $1 \leq i \leq m$ with $\sum_{i=1}^{m} d(i)=n$ then we denote by $\left[\lambda^{i}\right]_{i=1}^{m} \in \mathbb{R}^{n}$ the vector obtained by juxtaposition of the vectors $\lambda^{i}$ s s i.e $\lambda=\left(\lambda_{1}^{1}, \ldots, \lambda_{d(1)}^{1}, \lambda_{1}^{2}, \ldots, \lambda_{d(m)}^{m}\right)$.

Theorem 3.6 (NC Schur-Horn). Let $\mathbf{l}=(d(i), c(i))_{i=1}^{m} \in\left(\mathbb{N}^{2}\right)^{m}$ be such that $\sum_{i=1}^{m} d(i) \cdot c(i)=n$ and consider the unital $*$-subalgebra $\mathcal{A}=\oplus_{i=1}^{m} \mathcal{M}_{d(i)}(\mathbb{C}) \otimes 1_{c(i)} \subseteq \mathcal{M}_{n}(\mathbb{C})$. Let $\mathcal{E}_{\mathcal{A}}$ denote the trace preserving conditional expectation onto $\mathcal{A}$.

(i) If $B \in \mathcal{M}_{n}(\mathbb{C})^{\text {sa }}$ then there exists $M_{B}(\mathcal{A}) \subset \mathbb{R}^{n}$, that can be generated in terms of HornKlyachko's compatibility inequalities, 1 and $\lambda(B)$, such that

$$
\mathcal{E}_{\mathcal{A}}\left(\mathcal{U}_{n}(B)\right)=\left\{\oplus_{i=1}^{m} A_{i} \otimes 1_{c(i)} \in \mathcal{A}:\left[\lambda\left(A_{i} \otimes 1_{c(i)}\right)\right]_{i=1}^{m} \in M_{B}(\mathcal{A})\right\} .
$$

(ii) If $B \in \mathcal{M}_{n}(\mathbb{C})^{+}$then there exists $M_{B}^{w}(\mathcal{A}) \subset\left(\mathbb{R}_{\geq 0}\right)^{n}$, that can be generated in terms of Horn-Klyachko's compatibility inequalities, $\mathbf{l}$ and $\lambda(B)$, such that

$$
\mathcal{E}_{\mathcal{A}}\left(\mathcal{C}_{n}(B)\right)=\left\{\oplus_{i=1}^{m} A_{i} \otimes 1_{c(i)} \in \mathcal{A}:\left[\lambda\left(A_{i} \otimes 1_{c(i)}\right)\right]_{i=1}^{m} \in M_{B}^{w}(\mathcal{A})\right\} .
$$

Proof. Let us define $c=\sum_{i=1}^{m} c(i) \in \mathbb{N}$ and let $\mathbf{k}=(k(i))_{i=1}^{c}$ be the list given by

$$
k\left(\sum_{r=1}^{i-1} c(r)+j\right)=d(i) \text { for } 1 \leq i \leq m, \quad 1 \leq j \leq c(i) .
$$

We define first $D_{B}(\mathcal{A})$ as the set containing all $c$-tuples $\left(\mu^{i}\right)_{i=1}^{c}$ with $\mu^{i} \in \mathbb{R}^{k(i)}, \mu^{i}=\left(\mu^{i}\right)^{\downarrow}$ for $1 \leq i \leq c$ and such that the $(c+1)$-tuple

$$
\left(\lambda(B)+\|B\| \cdot \mathbf{e}^{n},\left(\mu^{1}+\|B\| \cdot \mathbf{e}^{k(1)}, 0_{n-k(1)}\right), \ldots,\left(\mu^{c}+\|B\| \cdot \mathbf{e}^{k(c)}, 0_{n-k(c)}\right)\right) \in\left(\mathbb{R}_{\geq 0}^{n}\right)^{c}
$$

and it satisfies Horn-Klyachko's compatibility inequalities plus $\operatorname{tr}(B)=\sum_{i=1}^{c} \sum_{j=1}^{k(i)} \mu_{j}^{i}$. If we let $\mathcal{P}=\left\{P_{i}\right\}_{i=1}^{c}$ be the system of coordinate projections with $\operatorname{rank}\left(P_{i}\right)=k(i)$ for $1 \leq i \leq c$ then, by Lemma 3.2, $\left(\mu^{i}\right)_{i=1}^{c} \in D_{B}(\mathcal{A})$ if and only if it can be realized as $\mu^{i}=\lambda\left(S_{i}\right)$ for $1 \leq i \leq c$, where $\mathcal{C}_{\mathcal{P}}\left(F^{*} B F\right)=\bigoplus_{i=1}^{c} S_{i}$ for some $F \in \mathcal{U}(n)$.

We now define $N_{B}(\mathcal{A})$ as the set containing all $\lambda=\left(\lambda^{i}\right)_{i=1}^{c}$, where $\lambda^{i}=\left(\lambda^{i}\right)^{\downarrow} \in \mathbb{R}^{k(i)}$ for $1 \leq i \leq c$ for which there exists $\left(\mu^{i}\right)_{i=1}^{c} \in D_{B}(\mathcal{A})$ such that, if $t(i)=\sum_{j=1}^{i-1} c(j)+1$ for $1 \leq i \leq m$ then

a) $\lambda^{t(i)}=\lambda^{t}$ for every $t(i) \leq t \leq t(i+1)-1$.

b) For $1 \leq i \leq m$ the $(c(i)+1)$-tuples (note that $k(t(i))=d(i))$

$$
\left(c(i) \lambda^{t(i)}, \mu^{t(i)}, \ldots, \mu^{t(i+1)-1}\right) \in\left(\mathbb{R}^{d(i)}\right)^{c(i)+1}
$$

satisfy Horn-Klyachko's compatibility inequalities plus the condition

$$
c(i) \sum_{j=1}^{d(i)} \lambda_{j}^{t(i)}=\sum_{j=t(i)}^{t(i+1)-1} \sum_{r=1}^{d(i)} \mu_{r}^{j} .
$$

Finally, we define $M_{B}(\mathcal{A})$ as the set containing all vectors $\eta=\left[\eta^{i}\right]_{i=1}^{m}$ where $\eta^{i} \in \mathbb{R}^{c(i) d(i)}$ for $1 \leq i \leq m$ and such that there exists $\lambda=\left(\lambda^{j}\right)_{j=1}^{c} \in N_{B}(\mathcal{A})$ with $\eta^{i}=\left[\lambda^{t(i)}, \ldots, \lambda^{t(i+1)-1}\right]^{\downarrow}$ for $1 \leq i \leq m$.

Now we show that if $A=\oplus_{i=1}^{m} A_{i} \otimes 1_{c(i)} \in \mathcal{A}$ is such that $\left[\lambda\left(A_{i} \otimes 1_{c(i)}\right)\right]_{i=1}^{m} \in M_{B}(\mathcal{A})$ then there exists a unitary matrix $U \in \mathcal{M}_{n}(\mathbb{C})$ such that $A=\mathcal{E}_{\mathcal{A}}\left(U^{*} B U\right)$. Recall that in this case the TCE onto $\mathcal{A}=\oplus_{i=1}^{m} \mathcal{M}_{d(i)}(\mathbb{C}) \otimes 1_{c(i)}$ is given by

$$
\mathcal{E}_{\mathcal{A}}(X)=\oplus_{i=1}^{m} \frac{1}{c(i)} \sum_{j=t(i)}^{t(i+1)-1} X_{j} \otimes 1_{c(i)}, \quad \text { with } \quad \mathcal{C}_{\mathcal{P}}(X)=\oplus_{i=1}^{c} X_{i}
$$

where $\mathcal{P}=\left\{P_{i}\right\}_{i=1}^{c}$ is as before. For $1 \leq i \leq m$ and $t(i) \leq j \leq t(i+1)-1$ let us define $\lambda^{j}:=\lambda\left(A_{i}\right)$ and let $\lambda:=\left(\lambda^{j}\right)_{j=1}^{c}$. By hypothesis there exists $\mu=\left(\mu^{i}\right)_{i=1}^{c} \in D_{B}(\mathcal{A})$ such that $(9)$ and $(10)$ hold for $\lambda$ and $\mu$. As remarked before, in this case there exists a unitary $F \in \mathcal{U}(n)$ such that 
$\mathcal{C}_{\mathcal{P}}\left(F^{*} B F\right)=\sum_{i=1}^{c} S_{i}$ and $\lambda\left(S_{i}\right)=\mu^{i}$ for $1 \leq i \leq c$. By condition b) and Theorem 2.1, for $1 \leq i \leq m$ there exist unitaries $W_{i, t(i)}, \ldots, W_{i, t(i+1)-1} \in \mathcal{U}(d(i))$ such that

$$
c(i) A_{i}=\sum_{j=t(i)}^{t(i+1)-1} W_{i, j}^{*} S_{i} W_{i, j}
$$

If we now define $W=\oplus_{i=1}^{m} \oplus_{j=t(i)}^{t(i+1)-1} W_{i, j} \in \mathcal{U}(n)$ then, by (12) we have

$$
\mathcal{C}_{\mathcal{P}}\left(W^{*} F^{*} B F W\right)=W^{*} \mathcal{C}_{\mathcal{P}}\left(F^{*} B F\right) W=\oplus_{i=1}^{m} \oplus_{j=t(i)}^{t(i+1)-1} W_{i, j}^{*} S_{i} W_{i, j}
$$

and hence, using a) above, (11) and (13) we get

$$
\mathcal{E}_{\mathcal{A}}\left(W^{*} F^{*} B F W\right)=\oplus_{i=1}^{m} \frac{1}{c(i)} \sum_{j=t(i)}^{t(i+1)-1} W_{i, j}^{*} S_{i} W_{i, j} \otimes 1_{c(i)}=A .
$$

On the other hand, if $\oplus_{i=1}^{m} A_{i} \otimes 1_{c(i)}=\mathcal{E}_{\mathcal{A}}\left(U^{*} B U\right)$ it is clear that $\left[\lambda\left(A_{i} \otimes 1_{c(i)}\right)\right]_{i=1}^{m} \in M_{B}(\mathcal{A})$. The second claim in (i) follows from Lemma 3.5 and the previous arguments.

To prove (ii), we proceed in a similar way using Lemma 3.4. We first define $\mathcal{D}_{B}^{w}(\mathcal{A})$ as the set containing all $c$-tuples $\left(\mu^{i}\right)_{i=1}^{c}$ with $\mu^{i} \in\left(\mathbb{R}_{\geq 0}\right)^{k(i)}, \mu^{i}=\left(\mu^{i}\right)^{\downarrow}$ for $1 \leq i \leq c$ and such that the $(c+1)$-tuple

$$
\left(\lambda(B),\left(\mu^{1}, 0_{n-k(1)}\right), \ldots,\left(\mu^{c}, 0_{n-k(c)}\right)\right)
$$

satisfies Horn-Klyachko's compatibility inequalities plus $\operatorname{tr}(B) \geq \sum_{i=1}^{c} \sum_{j=1}^{k(i)} \mu_{j}^{i}$. Then $N_{B}^{w}(\mathcal{A})$ and $M_{B}^{w}(\mathcal{A}) \subseteq\left(\mathbb{R}_{\geq 0}\right)^{n}$ are defined in terms of $\mathcal{D}_{B}^{w}(\mathcal{A})$ also using the conditions a) and b). The interested reader can now check that $M_{B}^{w}(\mathcal{A})$ has the desired properties following a similar argument to that above.

It seems difficult to describe formally the meaning of the assertion "that can be generated in terms of Horn-Klyachko's compatibility inequalities" in the statement of Theorem 3.6; but this should be clear after a careful inspection of the proof of this result.

Remark 3.7. Notice that in case $\mathcal{A}$ is the maximal abelian subalgebra of $\mathcal{M}_{n}(\mathbb{C})$ of (complex) diagonal matrices with respect to the canonical basis, the set $M_{B}(\mathcal{A})$ is already closed by permutation for any $B \in \mathcal{M}_{n}(\mathbb{C})^{s a}$. That is, for every $\sigma \in \mathbb{S}_{n}$ then $\lambda_{\sigma}=\left(\lambda_{\sigma(i)}\right)_{i=1}^{n} \in M_{B}(\mathcal{A})$ if and only if $\lambda=\left(\lambda_{i}\right)_{i=1}^{n} \in M_{B}(\mathcal{A})$. To see this last claim note that if $P_{\sigma}$ is the permutation matrix associated with $\sigma \in \mathbb{S}_{n}$ and $B \in \mathcal{M}_{n}(\mathbb{C})^{s a}$ then

$$
\mathcal{E}_{\mathcal{A}}\left(P_{\sigma}^{*} U^{*} B U P_{\sigma}\right)=P_{\sigma} \mathcal{E}_{\mathcal{A}}\left(U^{*} B U\right) P_{\sigma}
$$

where $\mathcal{E}_{\mathcal{A}}\left(U^{*} B U\right)$ is now a diagonal matrix.

Corollary 3.8. Let $\mathbf{l}=(d(i), c(i))_{i=1}^{m} \in\left(\mathbb{N}^{2}\right)^{m}$ be such that $\sum_{i=1}^{m} d(i) \cdot c(i)=n$. Using the notations of the NC-Schur-Horn theorem we have

(i) Given $A, B \in \mathcal{M}_{n}(\mathbb{C})^{\text {sa }}$, there exist unitary matrices $U, V \in \mathcal{U}(n)$ such that $U^{*} A U=$ $\mathcal{E}_{\mathcal{A}}\left(V^{*} B V\right)$ if and only if $\lambda(A) \in M_{B}(\mathbf{l}):=\left\{\mu^{\downarrow}: \mu \in M_{B}(\mathcal{A})\right\}$.

(ii) Given $A, B \in \mathcal{M}_{n}(\mathbb{C})^{+}$, there exist $U, V \in \mathcal{M}_{n}(\mathbb{C})$ with $U \in \mathcal{U}(n)$ and $\|V\| \leq 1$ such that $U^{*} A U=\mathcal{E}_{\mathcal{A}}\left(V^{*} B V\right)$ if and only if $\lambda(A) \in M_{B}^{w}(\mathbf{l})=\left\{\mu^{\downarrow}: \mu \in M_{B}^{w}(\mathcal{A})\right\}$.

\section{Extended Majorization in $\mathcal{M}_{n}(\mathbb{C})^{s a}$}

Ando extended in [1] the notion of vector (sub)majorization to that of (sub)majorization between elements in $\mathcal{M}_{n}(\mathbb{C})^{s a}$ i.e. the real vector space of hermitian matrices. Indeed, given $A, B \in$ $\mathcal{M}_{n}(\mathbb{C})^{s a}$ we say that $A$ is majorized (resp submajorized) by $B$, denoted $A \prec B\left(\operatorname{resp} A \prec_{w} B\right.$ ) if $\lambda(A) \prec \lambda(B)\left(\operatorname{resp} \lambda(A) \prec_{w} \lambda(B)\right)$. In this section, using the previous results, we present a spectral relation between self-adjoint matrices that extends majorization in the sense of Ando. For other extensions of majorization, the so called joint majorizations, see [22]. 


\subsection{Definition of extended majorization and basic properties.}

Definition 4.1 (Extended majorization and submajorization). Let $A, B \in \mathcal{M}_{n}(\mathbb{C})^{\text {sa }}$ and let $\mathbf{l}=(d(i), c(i))_{i=1}^{m} \in\left(\mathbb{N}^{2}\right)^{m}$ such that $\sum_{i=1}^{m} d(i) \cdot c(i)=n$. We say that B 1-majorizes A, denoted $A \prec_{\mathbf{l}} B$ iff

$$
\mathcal{U}_{n}(A) \cap \mathcal{E}_{\mathcal{A}}\left(\mathcal{U}_{n}(B)\right) \neq \emptyset \quad \text { or equivalently } \quad A \in \mathcal{U}_{n}\left(\mathcal{E}_{\mathcal{A}}\left(\mathcal{U}_{n}(B)\right)\right)
$$

for any (and then every) unital $*$-subalgebra $\mathcal{A} \subseteq \mathcal{M}_{n}(\mathbb{C})$ with spectral list $\mathbf{l}$.

If we further assume that $A, B \in \mathcal{M}_{n}(\mathbb{C})^{+}$then we say that $B$ 1-submajorizes $A$, denoted $A \prec_{\mathbf{l}, w} B$ iff

$$
\mathcal{U}_{n}(A) \cap \mathcal{E}_{\mathcal{A}}\left(\mathcal{C}_{n}(B)\right) \neq \emptyset \quad \text { or equivalently } \quad A \in \mathcal{U}_{n}\left(\mathcal{E}_{\mathcal{A}}\left(\mathcal{C}_{n}(B)\right)\right)
$$

for any (and then every) unital $*$-subalgebra $\mathcal{A} \subseteq \mathcal{M}_{n}(\mathbb{C})$ with spectral list 1 .

Note that Lemma 3.5 is the statement that l-majorization and l-submajorization are actually well defined

It is implicit in Definition 4.1 that these notions are actually well defined up to equivalence of spectral lists: given $A, B \in \mathcal{M}_{n}(\mathbb{C})^{s a}\left(\operatorname{resp} A, B \in \mathcal{M}_{n}(\mathbb{C})^{+}\right)$and $\mathbf{l}=(d(i), c(i))_{i=1}^{m}$ with $\sum_{i=1}^{m} d(i) c(i)=n$ then $A \prec_{\mathbf{l}} B$ if and only if $A \prec_{\mathbf{l}(\sigma)} B\left(\operatorname{resp} A \prec_{\mathbf{l}, w} B\right.$ if and only if $\left.A \prec_{\mathbf{l}(\sigma), w} B\right)$ for any (every) $\sigma \in \mathbb{S}_{m}$, where $\mathbf{l}(\sigma)=(d(\sigma(i)), c(\sigma(i)))_{i=1}^{m}$.

Remark 4.2. As a consequence of the NC-Schur-Horn theorem and Corollary 3.8 we conclude that l-(sub)majorization is a spectral relation that can be described explicitly in terms of HornKlyachko's compatibility inequalities. On the other hand, majorization in $\mathcal{M}_{n}(\mathbb{C})^{s a}$ in the sense of Ando corresponds to $\mathbf{l}$-majorization for the list $\mathbf{l}=(1,1)_{i=1}^{n}$ and hence the $\mathbf{l}$-majorization is an extension of usual majorization.

We shall need the following notion of refinement between multiplicity free lists. Given $\mathbf{l}_{1}=$ $\left(d_{1}(i), 1\right)_{i=1}^{m}, \mathbf{l}_{2}=\left(d_{2}(i), 1\right)_{i=1}^{t}$ such that $\sum_{i=1}^{m} d_{1}(i)=\sum_{i=1}^{t} d_{2}(i)=n$ we say that $\mathbf{l}_{1}$ refines $\mathbf{l}_{2}$ if there exist unital $*$-subalgebras $\mathcal{A} \subseteq \mathcal{B} \subseteq \mathcal{M}_{n}(\mathbb{C})$ such that $\mathcal{A}$ has spectral list $\mathrm{l}_{1}$ and $\mathcal{B}$ has spectral list $\mathbf{l}_{2}$. It is clear that $\mathbf{l}_{1}$ refines $\mathbf{l}_{2}$ if and only if there exists a partition $\{D(i)\}_{i=1}^{t}$ of the set $\{1, \ldots, m\}$ such that

$$
\sum_{i \in D(k)} d_{1}(i)=d_{2}(k), \quad 1 \leq k \leq t .
$$

Notice that every multiplicity free list is refined by the spectral list of a maximal abelian *subalgebra of $\mathcal{M}_{n}(\mathbb{C})$.

Proposition 4.3. Let $\mathbf{l}_{1}=\left(d_{1}(i), 1\right)_{i=1}^{m}, \mathbf{l}_{2}=\left(d_{2}(i), 1\right)_{i=1}^{t}$ be multiplicity free lists such that $\sum_{i=1}^{m} d_{1}(i)=\sum_{i=1}^{t} d_{2}(i)=n$. If we assume that $\mathbf{l}_{1}$ refines $\mathbf{l}_{2}$ then $\mathbf{l}_{2}$-(sub)majorization implies $\mathbf{l}_{1}$-(sub)majorization. In particular, $\mathbf{l}_{1}-($ sub)majorization is a reflexive and antisymmetric relation modulo unitary equivalence.

Proof. Let $\mathcal{A} \subseteq \mathcal{B} \subseteq \mathcal{M}_{n}(\mathbb{C})$ be unital $*$-subalgebras with spectral lists $\mathbf{l}_{1}$ and $\mathbf{l}_{2}$ respectively. Let $\mathcal{E}_{\mathcal{A}}, \mathcal{E}_{\mathcal{B}}$ be the corresponding TCE onto $\mathcal{A}$ and $\mathcal{B}$. Notice that in this case we have $\mathcal{E}_{\mathcal{A}} \circ \mathcal{E}_{\mathcal{B}}=\mathcal{E}_{\mathcal{A}}$. Moreover, since $\mathcal{A}$ is multiplicity free then there exists a maximal abelian $*$-subalgebra of $\mathcal{M}_{n}(\mathbb{C})$, denoted by $\mathcal{D}$, such that $\mathcal{D} \subseteq \mathcal{A}$. If we denote by $\mathcal{E}_{\mathcal{D}}$ the TCE onto $\mathcal{D}$ then $\mathcal{E}_{\mathcal{A}} \circ \mathcal{E}_{\mathcal{D}}=\mathcal{E}_{\mathcal{D}}$.

Let $A, B \in \mathcal{M}_{n}(\mathbb{C})^{s a}$ and let $U, V \in \mathcal{U}(n)$ be such that $\mathcal{E}_{\mathcal{B}}\left(U^{*} B U\right)=V^{*} A V$. Without loss of generality, we can assume that $V^{*} A V \in \mathcal{D}$. Then,

$$
\mathcal{E}_{\mathcal{A}} \circ \mathcal{E}_{\mathcal{B}}\left(U^{*} B U\right)=\mathcal{E}_{\mathcal{A}}\left(V^{*} A V\right)=V^{*} A V
$$

since $V^{*} A V \in \mathcal{D}$. A similar argument shows the submajorization statement. As a consequence of the argument above, we conclude that $\mathbf{l}_{1}$-(sub)majorization implies $\mathbf{l}$-(sub)majorization, where $\mathbf{l}=$ $(1,1)_{i=1}^{n}$ i.e. usual majorization. This last fact implies the antisymmetry of $\mathbf{l}_{1}$-(sub)majorization.

It is clear that $\mathbf{l}$-(sub)majorization, for lists which are not multiplicity free, is not reflexive nor antisymmetric in general. Because of these facts, in what follows we shall focus l-(sub)majorization for multiplicity free lists. On the other hand, the question of transitivity of l-(sub)majorization for a general list is open. 
4.2. Extended submajorization and convex functions. Given self-adjoint matrices $A, B \in$ $\mathcal{M}_{n}(\mathbb{C})^{s a}$ we say that $A$ is spectrally dominated by $B$, denoted $A \lesssim B$, if $\lambda(B)_{i} \geq \lambda(A)_{i}$ for $1 \leq i \leq n$. In this context it is straightforward that, given $A, B \in \mathcal{M}_{n}(\mathbb{C})^{+}$then $A \lesssim B$ if and only if there exists a contraction $V \in \mathcal{M}_{n}(\mathbb{C})$ such that $V^{*} B V=A$; but note that this last equation is, by definition, $A \prec_{\mathbf{t}, w} B$ for the trivial list $\mathbf{t}=(n, 1)$. Any multiplicity free list $\mathbf{l}=(d(i), 1)_{i=1}^{m}$ with $\sum_{i=1}^{m} d(i)=n$, refines (as defined before Proposition 4.3) the list $\mathbf{t}$. Hence, by Proposition 4.3, we get the equivalence: for $A, B \in \mathcal{M}_{n}(\mathbb{C})^{+}$,

$$
A \lesssim B \text { if and only if } A \prec_{\mathbf{l}, w} B
$$

for every multiplicity free list $\mathbf{l}$ as above.

Proposition 4.4 (Jensen's inequality). Let $f:(\alpha, \beta) \rightarrow[0, \infty)$ be a monotone convex function and let $A \in \mathcal{M}_{n}(\mathbb{C})^{\text {sa }}$ be such that the spectrum of $A$ is contained in $(\alpha, \beta)$. Then, for every system of coordinate projections $\mathcal{P}=\left\{P_{i}\right\}_{i=1}^{t}$ and for every multiplicity free list $\mathbf{l}=(d(i), 1)_{i=1}^{m}$ with $\sum_{i=1}^{m} d(i)=n$

$$
f\left(\mathcal{C}_{\mathcal{P}}(A)\right) \prec_{\mathbf{1}, w} \mathcal{C}_{\mathcal{P}}(f(A)) .
$$

Proof. Let $f$ be a monotone convex function and let $\mathcal{P}$ be a system of coordinate projections as above. As a consequence of theorem 3.1 in [2] we get that $f\left(\mathcal{C}_{\mathcal{P}}(A)\right) \lesssim \mathcal{C}_{\mathcal{P}}(f(A))$. The result now follows from (14).

Let $f:[0, \infty) \rightarrow[0, \infty)$ be a convex function with $f(0)=0$, and hence non-decreasing. If $A, B \in \mathcal{M}_{n}(\mathbb{C})^{+}$are such that $A \prec_{w} B$ then [1] we have that $f(A) \prec_{w} f(B)$ i.e. $f$ is monotonic with respect to submajorization. The next result is a generalization of this fact to the context of l-submajorization for multiplicity free lists 1 .

Proposition 4.5. Let $f:[0, \infty) \rightarrow[0, \infty)$ be a convex function with $f(0)=0$ and let $\mathcal{P}=\left\{P_{i}\right\}_{i=1}^{m}$ be a system of coordinate projections with $\operatorname{rank}\left(P_{i}\right)=d(i), 1 \leq i \leq m$. Let $W \in \mathcal{M}_{n}(\mathbb{C})$ be such that $\|W\| \leq 1$ and $A, B \in \mathcal{M}_{n}(\mathbb{C})^{+}$be such that $\mathcal{C}_{\mathcal{P}}\left(W^{*} B W\right)=A$. Then, there exists $\tilde{W} \in \mathcal{M}_{n}(\mathbb{C})$ with $\|\tilde{W}\| \leq 1$ and such that $\mathcal{C}_{\mathcal{P}}\left(\tilde{W}^{*} f(B) \tilde{W}\right)=f(A)$.

Proof. Let $A, B \in \mathcal{M}_{n}(\mathbb{C})^{+}$be such that $A \prec_{1, w} B$ and let $f$ be as above. We assume that $\mathcal{C}_{\mathcal{P}}\left(W^{*} B W\right)=\oplus_{i=1}^{m} A_{i}=A$ for a contraction $W \in \mathcal{M}_{n}(\mathbb{C})$. We shall need the following result from [9]: if $X \in \mathcal{M}_{n}(\mathbb{C})$ is a contraction then there exists $V \in \mathcal{U}(n)$ such that $f\left(X^{*} B X\right) \leq$ $V^{*} X^{*} f(B) X V$. Fix $1 \leq i \leq m$. Using the previous result and the fact that for every $T \in \mathcal{M}_{n}(\mathbb{C})$ $T T^{*}$ and $T^{*} T$ are unitarily equivalent, we conclude that there there exist $U_{i}, V_{i} \in \mathcal{U}(n)$ such that

$$
f\left(\left(P_{i} W^{*}\right) B\left(W P_{i}\right)\right) \leq V_{i}^{*} P_{i} W^{*} f(B) W P_{i} V_{i}=U_{i}^{*} f(B)^{1 / 2} W P_{i} W^{*} f(B)^{1 / 2} U_{i} .
$$

Then

$$
\begin{aligned}
f(B) & \geq \sum_{i=1}^{m} f(B)^{1 / 2} W P_{i} W^{*} f(B)^{1 / 2} \\
& \geq \sum_{i=1}^{m} U_{i} f\left(P_{i} W^{*} B W P_{i}\right) U_{i}^{*}=\sum_{i=1}^{m} U_{i}\left(\oplus_{j=1}^{m} \delta_{i j} f\left(A_{i}\right)\right) U_{i}^{*}
\end{aligned}
$$

and the proposition now follows from Lemma 3.4.

Corollary 4.6. Let $\mathbf{l}=((d(i), 1))_{i=1}^{m}$ be a multiplicity free list with $\sum_{i=1}^{m} d(i)=n$. If $A, B \in$ $\mathcal{M}_{n}(\mathbb{C})^{+}$then the following statements are equivalent:

(i) $A \prec_{\mathbf{1}, w} B$.

(ii) For every convex function $f:[0, \infty) \rightarrow[0, \infty)$ with $f(0)=0$ we have $f(A) \prec_{1, w} f(B)$.

In particular, every convex function $f:[0, \infty) \rightarrow[0, \infty)$ with $f(0)=0$ is monotonic with respect to 1-submajorization.

The next result, which follows from our previous arguments, is theorem 2.1 in [8] expressed in terms of convex functions. Its proof illustrates the use of extended majorization.

Corollary 4.7. Let $f:[0, \infty) \rightarrow[0, \infty)$ be a convex function with $f(0)=0$ and let $A, B \in$ $\mathcal{M}_{n}(\mathbb{C})^{+}$. Then there exist unitary matrices $U, V \in \mathcal{U}(n)$ such that

$$
U^{*} f(A) U+V^{*} f(B) V \leq f(A+B) .
$$


Proof. Consider the $2 n \times 2 n$ matrices

$$
\left(\begin{array}{cc}
A+B & 0 \\
0 & 0
\end{array}\right)=\left(\begin{array}{ll}
A & 0 \\
0 & 0
\end{array}\right)+\left(\begin{array}{ll}
0 & 1 \\
1 & 0
\end{array}\right)\left(\begin{array}{ll}
0 & 0 \\
0 & B
\end{array}\right)\left(\begin{array}{ll}
0 & 1 \\
1 & 0
\end{array}\right)
$$

Let $\mathbf{l}=((n, 1),(n, 1))$. By item (ii) in Lemma 3.4 and Proposition 4.5 there exist unitary matrices $\tilde{U}, \tilde{V} \in \mathcal{U}(2 n)$ such that

$$
\tilde{U}^{*}\left(\begin{array}{cc}
f(A) & 0 \\
0 & 0
\end{array}\right) \tilde{U}+\tilde{V}\left(\begin{array}{cc}
0 & 0 \\
0 & f(B)
\end{array}\right) \tilde{V} \leq\left(\begin{array}{cc}
f(A+B) & 0 \\
0 & 0
\end{array}\right) .
$$

If $\tilde{U}=\left(U_{i j}\right)_{i j=1}^{2}$ then, by compressing (16) to the $(2,2)$ block we get $U_{12}^{*} f(A) U_{12}=0$ and hence $Q U_{12}=0$ where $Q$ is the projection onto the range of $f(A) \geq 0$. Therefore

$$
U_{11} U_{11}^{*}+U_{12} U_{12}^{*}=1_{n} \Rightarrow Q\left(U_{11} U_{11}^{*}\right) Q=Q .
$$

Thus, $Q U_{11} \in \mathcal{M}_{n}(\mathbb{C})$ is a partial isometry with the same range as $Q$. Therefore there exists $U \in \mathcal{U}(n)$ such that $Q U=Q U_{11}$, and then

$$
U^{*} f(A) U=U_{11}^{*} f(A) U_{11}=\left(\tilde{U}\left(\begin{array}{cc}
f(A) & 0 \\
0 & 0
\end{array}\right) \tilde{U}\right)_{11}
$$

where the sub-index 11 in the right-hand side of this last equation stands for the $(1,1)$-block. Similarly, there exists $V \in \mathcal{U}(n)$ such that

$$
V^{*} f(B) V=\left(\tilde{V}\left(\begin{array}{cc}
0 & 0 \\
0 & f(B)
\end{array}\right) \tilde{V}\right)_{11}
$$

The corollary now follows by compressing the inequality (16) to the (1,1)-block and using (17) and (18).

4.3. A non-commutative Horn's lemma and QIT. In [26] the following problem is posed in the context of Quantum Information Theory (QIT).

Conjecture 4.8 (from [26]). Let $A \in \mathcal{M}_{d \cdot m}(\mathbb{C})^{+}$be a block matrix $A=\left(A_{i j}\right)_{i, j=1}^{m}$ with $A_{i j} \in$ $\mathcal{M}_{d}(\mathbb{C})$ for $1 \leq i, j \leq m$ and let $M=\sum_{i=1}^{m} A_{i i} \in \mathcal{M}_{d}(\mathbb{C})^{+}$. Then there exist rectangular matrices $X_{i} \in \mathcal{M}_{d \cdot m, d}(\mathbb{C}), X_{i}^{*}=\left(X_{1 i}^{*}, \ldots, X_{m i}^{*}\right)$ with $X_{i j} \in \mathcal{M}_{d}(\mathbb{C})$ for $1 \leq i, j \leq m$ such that

$$
A=\frac{1}{m} \sum_{i=1}^{m} X_{i} X_{i}^{*} \quad \text { and } \quad \sum_{j=1}^{m} X_{j i} X_{j i}^{*}=M, 1 \leq i \leq m .
$$

The previous conjecture can be also expressed in terms of partial traces. We see that we can replace (19) in Conjecture 4.8 by

$$
A=\frac{1}{m} \sum_{i=1}^{m} X_{i} X_{i}^{*} \quad \text { and } \quad \operatorname{Tr}_{m}\left(X_{i} X_{i}^{*}\right)=\operatorname{Tr}_{m}(A), 1 \leq i \leq m .
$$

Conjecture 4.8 is related with certain convex decompositions of unital completely positive (UCP) maps between matrix algebras, in terms of Choi matrices, that are of interest in QIT. The case $d=1$ is solved in [26] using what is called "Horn's lemma" namely, that given $A \in \mathcal{M}_{n}(\mathbb{C})^{+}$with $\operatorname{tr}(A)=1$ there exist $U, B \in \mathcal{M}_{n}(\mathbb{C})$ with $U$ unitary, $B$ with diagonal entries all equal to $1 / n$ and $U^{*} A U=B$. The following result is an analogue of the above Horn's lemma which leads to a related representation to that in (19). Still, while the convex decomposition that is obtained using our result expresses a UCP map as an average of completely positive maps with Choi rank at most $m$, these representing maps may fail to be unital.

Proposition 4.9 (A non-commutative Horn's lemma). Let $A \in \mathcal{M}_{d \cdot m}(\mathbb{C})^{+}$be a block matrix $A=\left(A_{i j}\right)_{i, j=1}^{m}$ with $A_{i j} \in \mathcal{M}_{d}(\mathbb{C})$ for $1 \leq i, j \leq m$. Let $\mathcal{P}=\left\{P_{i}\right\}_{i=1}^{m}$ be a system of coordinate projections such that $\operatorname{rank}\left(P_{i}\right)=d, 1 \leq i \leq m$.

(i) There exists $U \in \mathcal{U}(d \cdot m)$ and $D \in \mathcal{M}_{d}(\mathbb{C})^{+}$such that

$$
\mathcal{C}_{\mathcal{P}}\left(U^{*} A U\right)=\frac{1}{m} \oplus_{i=1}^{m} D .
$$


(ii) There exist rectangular matrices $X_{i} \in \mathcal{M}_{d \cdot m, d}(\mathbb{C}), X_{i}^{*}=\left(X_{1 i}^{*}, \ldots, X_{m i}^{*}\right)$ with $X_{i j} \in$ $\mathcal{M}_{d}(\mathbb{C})$ and unitary matrices $U_{i} \in \mathcal{U}(d \cdot m)$ for $1 \leq i, j \leq m$, such that

$$
\begin{aligned}
& \qquad A=\frac{1}{m} \sum_{i=1}^{m} X_{i} X_{i}^{*} \quad \text { and } \quad U_{i}^{*} X_{i} X_{i}^{*} U_{i}=\oplus_{j=1}^{m} \delta_{i j} D, \\
& \text { and hence } \operatorname{Tr}_{m}\left(U_{i}^{*}\left(X_{i} X_{i}^{*}\right) U_{i}\right)=\operatorname{Tr}_{m}\left(U^{*} A U\right), \quad 1 \leq i \leq m .
\end{aligned}
$$

Proof. Note that (ii) is a direct consequence of (i) and Lemma 3.2 (with $\alpha=0$ ). Indeed, if we assume (i) then there exist $U_{i} \in \mathcal{U}(d \cdot m)$ for $1 \leq i \leq m$ such that

$$
A=\frac{1}{m} \sum_{i=1}^{m} U_{i}\left(\oplus_{j=1}^{m} \delta_{i j} D\right) U_{i}^{*}=\frac{1}{m} \sum_{i=1}^{m} X_{i} X_{i}^{*}
$$

where

with $U_{i}=\left(U_{l k}^{(i)}\right)_{l, k=1}^{m}$ and $U_{l k}^{(i)} \in \mathcal{M}_{d}(\mathbb{C})$.

$$
X_{i}^{*}=\left(D^{1 / 2}\left(U_{1 i}^{(i)}\right)^{*}, \ldots, D^{1 / 2}\left(U_{m i}^{(i)}\right)^{*}\right),
$$

To prove (i) consider first $\xi \in \mathbb{C}$ an $m$-th primitive root of unity and let $\tilde{V} \in \mathcal{M}_{m}(\mathbb{C})$ be the matrix with $j$-th row given by

$$
R_{j}(\tilde{V})=1 / \sqrt{m}\left(1, \xi^{j}, \xi^{2 j}, \ldots, \xi^{(m-1) j}\right), \quad 1 \leq j \leq m .
$$

It is then straightforward to show that the rows of $\tilde{V}$ form an orthonormal basis for $\mathbb{C}^{m}$ and hence $\tilde{V} \in \mathcal{U}(m)$ is a unitary matrix. Let $V \in \mathcal{U}(d \cdot m)$ be the block matrix $V=\left(\tilde{V}_{i j} \cdot 1_{d}\right)_{i, j=1}^{m}$. If $W \in \mathcal{U}(d \cdot m)$ is such that $W^{*} A W=\oplus_{i=1}^{m} D_{i}$ where $D_{i} \in \mathcal{M}_{d}(\mathbb{C})$ is a diagonal matrix $1 \leq i \leq m$, define $U:=V W$ and note that

$$
\mathcal{C}_{\mathcal{P}}\left((W V)^{*} A(W V)\right)=\mathcal{C}_{\mathcal{P}}\left(V^{*}\left(\oplus_{i=1}^{m} D_{i}\right) V\right)=\frac{1}{m} \oplus_{i=1}^{m}\left(\sum_{j=1}^{m} D_{j}\right)
$$

The last equality follows from the block structure of $\oplus_{i=1}^{m} D_{i}$ and by construction of $V$. Thus, we define $D:=\sum_{j=1}^{m} D_{j}$.

Note that the particular case $d=1$ of Conjecture 4.8 follows from Proposition 4.9 , since $\operatorname{Tr}_{m}=$ tr in this case. But we remark that the general case of Conjecture 4.8 does not follow from Proposition 4.9, since the equation $\operatorname{Tr}_{m}\left(U^{*} A U\right)=\operatorname{Tr}_{m}\left(U_{i}^{*} X_{i} X_{i}^{*} U_{i}\right)$ does not imply (for $d>1$ ) that $\operatorname{Tr}_{m}\left(X_{i} X_{i}^{*}\right)=\operatorname{Tr}_{m}(A)$. This is a consequence of the non-commutativity of the values of $\operatorname{Tr}_{m}$.

Also notice that the matrix $D$ above is not unique. Moreover, there does not seem to be a canonical choice of $D$ in general. Hence, if we let $\mathbf{d}=(d, \ldots, d) \in \mathbb{R}^{m}$, it is not clear whether there is in general a minimum (up to unitary equivalence) with respect to d-majorization of the set $\left\{A \in \mathcal{M}_{d \cdot m}(\mathbb{C})^{+}: \operatorname{Tr}_{m}(A)=1\right\}$.

Remark 4.10. It is worth noting that the case $m=2$ of the Conjecture 4.8 has been proved (see [26]). But the ideas involved in the proof are related with the off-diagonal blocks of the $2 \times 2$ representation of $A$.

Acknowledgments. This note is the consequence of a talk I gave in the Canadian Operator Symposium (COSy) at Guelph. For that I would like to thank the organizers J. Holbrook and D. Kribs, and the Fields Institute for funding support to attend this event. I would also like to thank the people in the Math and Stats department at the University of Regina for their kind hospitality during my PIMS pdf there, particularly to M. Argerami, D. Farenick and S. Fallat.

\section{REFERENCES}

[1] T. Ando, Majorization, doubly stochastic matrices and comparison of eigenvalues, Lecture Notes, Hokkaido Univ., 1982.

[2] J. Antezana, P. Massey, and D. Stojanoff, Jensen's Inequality and Majorization, J. Math. Anal. Appl 331 (2007) 297-307.

[3] J. Antezana, P. Massey, M. Ruiz, and D. Stojanoff, The Schur-Horn theorem for operators and frames with prescribed norms and frame operator, Illinois J. of Math 51(2) (2007) 537-560.

[4] M. Argerami and P. Massey, A Schur-Horn theorem for II 1 factors, Indiana Univ. Math. J. 56(5) (2007) 2051-2059. 
[5] W. Arveson and R. Kadison, Diagonals of self-adjoint operators, In D. R. Larson D. Han, P. E. T. Jorgensen, editor, Operator theory, operator algebras and applications, Contemp. Math. Amer. Math. Soc., 2006 arXiv:math.OA/0508482 v2.

[6] M. Atiyah, Convexity and commuting Hamiltonians, Bull. L.M.S., 14(1) (1982) 1-15.

[7] R. Bhatia, Partial traces and entropy inequalities, Linear Algebra Appl. 370 (2003) 125-132.

[8] J.S. Aujla, J.C. Bourin, Eigenvalue inequalities for convex and log-convex functions, Linear Algebra Appl. 424 (2007) 25-35.

[9] J.S. Aujla, F.C. Silva, Weak majorization inequalities and convex functions, Linear Algebra Appl. 369 (2003) 217-233.

[10] I.S. Dhillon, R.W. Heath Jr.,M.A. Sustik, J.A. Tropp, Generalized finite algorithms for constructing Hermitian matrices with prescribed diagonal and spectrum SIAM J. Matrix Anal. Appl. 27 (2005) 61-71.

[11] S. Friedland, Finite and infinite dimensional generalizations of Klyachko's theorem, Linear Algebra Appl. 319 (2000) 3-22.

[12] W. Fulton, Eigenvalues of majorized Hermitian matrices and Littlewood-Richardson coefficients, Linear Algebra Appl. 319 (2000) 23-36.

[13] W. Fulton, Eigenvalues, invariant factors, highest weights, and Schubert calculus, Bull. Amer. Math. Soc. 37 (2000) 209-249, math.AG/9908012.

[14] V. Guillemin and S. Sternberg Convexity properties of the moment mapping, Invent. Math., 67 (3) (1982) 491-513.

[15] A. Horn. Doubly stochastic matrices and the diagonal of a rotation matrix. Amer. J. Math., 76(3) (1954), 620-630.

[16] R. Kadison, The Pythagorean Theorem: I. The finite case, PNAS 99(7), 2002, 4178-4184.

[17] R. Kadison, The Pythagorean Theorem: II. The infinite discrete case, PNAS 99(8), 2003, 5217-5222.

[18] A.A. Klyachko, Stable bundles, representation theory and Hermitian operators, Selecta Math. 4 (1998) 419-445.

[19] B. Konstant, On convexity, the Weyl group and the Iwasawa decomposition, Ann. Scient. Éc. Norm. Sup., 6: (1973) 413-455.

[20] R.S. Leite, T.R.W. Richa and C. Tomei, Geometric proofs of some theorems of Schur-Horn type, Linear Algebra Appl. 286 (1999) 149-173.

[21] C.K. Li and Y.T. Poon,Principal submatrices of a Hermitian matrix, Linear Multilinear Algebra 51(2) (2003), 199-208.

[22] F. Martínez Pería, P. Massey and L. Silvestre, Weak matrix majorization, Linear Algebra Appl. 403 (2005), 343-368.

[23] P. Massey, Optimal reconstruction systems for erasures and for the q-potential, submitted.

[24] P. Massey and M. Ruiz, Some convex functionals over frame operators, Adv. Comp. Math, in press.

[25] A. Neumann, An infinite-dimensional version of the Schur-Horn convexity theorem, J. Funct. Anal. 161 (1999), 418-451.

[26] M.B. Ruskai, Some open problems in QIT, preprint.

[27] I. Schur, Über eine klasse von mittlebildungen mit anwendungen auf der determinantentheorie, Sitzungsber. Berliner Mat. Ges., 22 (1923), 9-29. 